\title{
Exploring the limits of spatial memory in rats, using very large mazes
}

\author{
MARK R. COLE and ROBYN CHAPPELL-STEPHENSON \\ Huron University College, London, Ontario, Canada
}

\begin{abstract}
In Experiment 1, rats foraged for food in six successive phases with 8, 16, 24, 32, 40, and 48 arms attached in random locations to a large radial maze. The percentage of novel choices appeared to be determined more by spatial proximity than by number of arms. In Experiment 2, rats foraged for food in four successive phases with $8,16,24$, and 48 arms attached to the maze in spread-out or tight configurations. Performance was poor in the tight configurations regardless of the number of arms. Performance was excellent in the 8-arm spread-out condition but declined as 16 and, then again, 24 arms were added. Thus, spatial separation, not number of locations, was the chief determinant of performance in the first two experiments. In Experiment 3, in successive phases, 8, 16, 24, 32, 40, 48, 16, and 8 food towers were set in a circle on the floor, with the spatial separation between adjacent towers held constant at $33 \mathrm{~cm}$. The percentage of novel choices declined as 8 towers became 16 and did not change again with $24,32,40$, or 48 towers in place but then increased again as 16 towers became 8 . In Experiment 4 , in successive phases, $8,16,24$, and 32 food towers were set in a circle, with the spatial separation between adjacent towers held constant at $66 \mathrm{~cm}$. The percentage of novel choices declined as 8 towers became 16 and again as 16 towers became 24 but did not decline further. These data were discussed in terms of the fundamental problems posed by variations in the number of food locations in the pursuit of the limit of spatial memory in rats.
\end{abstract}

Since the publication of the seminal work by Olton and Samuelson (1976), which showed that rats have excellent spatial memory for food locations visited on a radial maze, there has been a great deal of research carried out using this apparatus. In view of that, it is surprising that more attention has not been devoted to testing the limits of spatial memory in rats with the radial maze. Olton and Samuelson showed that on the original 8-arm maze, rats made about $87 \%$ novel entries in their first eight attempts. Olton, Collison, and Werz (1977) reported that when the maze was extended to 17 arms, correct choices declined slightly to about $82 \%$. Whether this is a statis-

Portions of the present research were presented at various conferences, including the meetings of the Canadian Society for Brain, Behaviour and Cognitive Sciences in Edmonton, June 1999; the joint meeting of the Canadian Society for Brain, Behaviour and Cognitive Sciences and the Experimental Psychology Society in Cambridge, England, July 2000; the meetings of the Canadian Society for Brain, Behavior and Cognitive Sciences in Quebec City, June 2001; and the meetings of the Canadian Society for Brain, Behavior and Cognitive Sciences in Vancouver, June 2002. This research was supported by grants from the Faculty of Arts and Social Sciences at Huron University College. Much gratitude is due Kathy Bracken, Ken Bignell, Amy Clipperton, Nadine Magid, and Dana Tobe, all of whom assisted in data collection and/or analysis. We thank Stephen Erdle for advice regarding analysis and the entire Psychology 501 Graduate Group for advice and counsel, with special thanks to Nancy Innis for her helpful suggestions. Finally, thanks to Lori Cole for cheerfully accepting all those undone household chores over four summers. Correspondence concerning this article should be directed to M. R. Cole, Department of Psychology, Huron University College, 1349 Western Road, London, ON, N6G 1H3 Canada (e-mail: mcole@uwo.ca). tically significant difference is not known. Roberts (1979) used a hierarchical radial maze, with each of 8 primary arms splitting off into 3 secondary arms, for a total of 24 food locations. He tested rats with just 1 , just 2 , or all 3 of the secondary arms at the end of each primary arm open to the rat. He found no differences in the percentages of errors in any of the conditions. Strictly speaking, however, this arrangement is not directly comparable to the previous research by Olton and Samuelson and by Olton et al. With the hierarchical maze, memory for food locations might have been improved by chunking. That is, the memory load might have been eased when the rats, having recalled which primary arm had been entered, had only then to recall which secondary arm to enter. Research both with humans (e.g., Tulving \& Pearlstone, 1966) and with rats (e.g., Dallal \& Meck, 1990) has shown that memory is improved when the material to be remembered can be categorized or chunked.

The experiments reported herein were designed to explore further the effect that increasing the number of food locations beyond 16 has on foraging efficiency by rats in nonhierarchical settings.

\section{EXPERIMENT 1}

Experiment 1 employed a radial maze, with a very large central platform capable of supporting up to 48 arms. The aim was to explore more fully the question of how spatial memory fares when the number of arms on the maze is systematically increased. The basic approach 
was to add 8 arms at a time to randomly selected locations on the maze, to see what effect this would have on performance.

\section{Method}

\section{Subjects}

Five male hooded rats of the Long-Evans strain, obtained from Charles River in Montreal and with prior experience both in operant chambers and on a small eight-arm radial maze, were used. The rats were reduced to $85 \%$ of their free-feeding body weight and were housed individually in 2-rat shoe box style cages with water available ad lib. The cage room was maintained at $23^{\circ} \mathrm{C}$ with a 12:12-h light:dark cycle. The lights were red during the first and last hours of the lighted period. Testing was carried out during the light-on phase. The rats in this and the subsequent experiments were treated at all times in accordance with the Ethical Guidelines of the Canadian Council on Animal Care.

\section{Apparatus}

The maze was constructed of 1.9-cm-thick poplar plywood and consisted of a circular central platform, $152 \mathrm{~cm}$ in diameter, and 48 arms, each $61 \mathrm{~cm}$ long $\times 9.5 \mathrm{~cm}$ wide. Each arm was attached to the central platform by means of a pair of parallel steel mending plates set $6.0 \mathrm{~cm}$ apart and attached to the upper surface of the maze. Each mending plate was secured by means of two $2.0-\mathrm{cm}-$ long wood screws, one screwed into the arm and one into the central platform. A plastic food cup created from a 35-mm film canister, $2.5 \mathrm{~cm}$ in diameter and $1.9 \mathrm{~cm}$ deep, was attached by means of a small screw at a point $2.5 \mathrm{~cm}$ from the end of each arm and equidistant from the sides of the arm. Screwed to the sides of each arm were triangular sidewalls made of $0.5-\mathrm{cm}$-thick foam core and designed to discourage arm-to-arm traverses. The side walls rose $30.5 \mathrm{~cm}$ above the surface of the arm at the food cup end of each arm but sloped down so that they were $0 \mathrm{~cm}$ above the surface of the arm at a point $40.5 \mathrm{~cm}$ from the food cup end of the arm. Thus, there was no wall at all attached to the first $20.5 \mathrm{~cm}$ of each arm, measured from the edge of the central platform. The arms, when all were attached, were equidistant from each other, and adjacent edges were about $8.0 \mathrm{~cm}$ apart at the food cup end and $0.5 \mathrm{~cm}$ apart where they joined the central platform. A pair of sturdy tables located entirely under the central platform supported the maze so that the surface of the maze was $76 \mathrm{~cm}$ above the floor. The upper surface of the central platform and both the upper surface and the sides and ends of the arms were painted with white high-gloss enamel paint. White paint was used to make it easier for the observer to identify the location of the rat in the maze. The maze was located in a room measuring $4.5 \times 5.0 \mathrm{~m}$. The northeast corner of the room had an alcove beyond this perimeter, which measured $1.5 \times 0.6 \mathrm{~m}$ and served as a location for the experimenter to occupy while recording data. There was at least one door on each wall, except for the west wall, which was the location of a stainless steel sink and a table. Arm locations were numbered clockwise, starting with the most easterly location. A $3.0 \mathrm{~cm} \times 3.0 \mathrm{~cm}$ Post-it note with a black dot $0.25 \mathrm{~cm}$ in diameter in its center was fixed to the outside of one of the sidewalls attached to any arm in Location 1, 7, 13, 19, 25, 31, 37 , or 43 . In addition, a $3.0 \times 3.0 \mathrm{~cm}$ magenta Post-it note was fixed to one of the sidewalls attached to any arm in Location 4, 10, 16, 22, $28,34,40$, or 46 . Since the purpose of these markers was to assist the researchers in accurately recording arm entries, all the markers were attached in such a way that they were visible to the experimenters as they recorded data. Although the markers were also visible to the rats, the researchers could detect no evidence that they made use of them in any way.

Formula A/I Dustless Food Pellets, each weighing $45 \mathrm{mg}$ and manufactured by the Noyes Company, were used to bait the food cups.

\section{Procedure}

The locations for the eight arms used in the first phase were randomly selected, and arms were attached to those locations. In successive phases, additional sets of 8 locations were randomly selected, and arms were added to those locations until, by the sixth phase, all 48 arm locations had been selected and all the arms had been added. Because it would not have been possible to maintain symmetrical arrangements on the maze with 32 or 40 arms, a decision was made to assign the arm locations randomly from the outset. The arm locations thus selected in each phase are shown in Table 1.

Preliminary training. With the first eight arms attached, the rats were given five trials of preliminary training in order to familiarize them with the maze. By the end of this preliminary training, the rats had visited all the arms on a trial and had eaten from the food cups. On these and on subsequent experimental trials, the maze was washed with a mild soap solution, all arms were baited with a single Noyes pellet, and the rat was placed as close as possible to the center of the central platform, always facing west. The rat remained in the maze until it met one of several criteria, described below.

Training proper. Following preliminary training, the rats were given two trials per day, with the first daily trial session starting at about 8 a.m. and the second beginning at about 12 p.m. The rat remained in the maze until it reached criterion, as described below. After being removed from the maze in the afternoon session, the rat was weighed and given a ration of lab chow sufficient to maintain its body weight at $85 \%$ of the original free-feeding weight. After each set of 10 trials, an additional eight arms were added to the maze.

Trial-ending criteria. With $n$ referring to the number of arms on the maze at the time, the rat remained in the maze until it met one of the following criteria: (1) $n$ choices had been made, (2) $n$ minutes had passed, or (3) the rat had remained for at least 3 min in any one arm.

Data recording. After placing the rat in the maze, the researcher retreated to a chair positioned on top of a table in the alcove. This elevated vantage point permitted the observer to see all parts of the maze clearly. Facsimiles of the maze were used to record the order of arm entries.

Satiation test. The same 5 rats were tested in this phase, and they were maintained on the same $85 \%$ food deprivation schedule as that used in the first part of this experiment. A table measuring $1.54 \times$ $.77 \mathrm{~m}$ and standing $77 \mathrm{~cm}$ above the floor was used in place of the radial maze. The table was located in a small test room measuring $2.9 \times 2.1 \mathrm{~m}$. The left rear corner of the table was pressed into the left rear corner of the room. Two daily sessions were run for each rat, the first in the morning and the second in the afternoon. At the start of each session, 48 Noyes pellets, each weighing $45 \mathrm{mg}$, were scattered in a small circle measuring about $30 \mathrm{~cm}$ in diameter, the circumference of which was about $30 \mathrm{~cm}$ from each of the walls enclosing the left rear corner of the table. The rat was placed on the table near the food pellets, and the time it took to eat the first and second sets of 24 pellets was recorded. When all pellets had been

Table 1

Arm Locations in Each of the Six Successive Phases in Experiment 1

\begin{tabular}{crrrrrrrr}
\hline Phase & \multicolumn{7}{c}{ Selected Locations } \\
\hline 1 & 5 & 10 & 11 & 16 & 19 & 36 & 39 & 46 \\
2 & 6 & 14 & 20 & 21 & 32 & 41 & 42 & 48 \\
3 & 2 & 17 & 18 & 22 & 23 & 31 & 35 & 38 \\
4 & 8 & 12 & 13 & 15 & 24 & 25 & 28 & 44 \\
5 & 3 & 4 & 7 & 9 & 26 & 33 & 34 & 40 \\
6 & 1 & 27 & 29 & 30 & 37 & 43 & 45 & 47 \\
\hline
\end{tabular}


consumed, the rat was removed. The table was washed with a diluted soap solution after each trial and before the next rat was tested. In all, six sessions were carried out, with the data from the last five being analyzed.

\section{Results}

\section{Memory for Locations}

The chief dependent variable of interest in the present experiment was percentage of novel entries in the first $n$ opportunities (where $n$ was the number of arms attached to the maze). The use of percentages enabled a fair comparison of the results from conditions involving different numbers of arms. These data are shown in Figure 1. To facilitate interpretation, an estimate of chance performance on the maze is also shown for each value of $n$. This estimate of chance performance is the expected number of novel entries in the first $n$ opportunities, assuming random sampling with replacement. First, for each value of $n$, the probability of being correct on successive choices from 1 to $n$ was calculated from the formula $[(n-1) / n]^{r-1}$ (Bond, Cook, \& Lamb, 1981), in which $n$ was the number of arms attached to the maze and $r$ was the choice being made. The sum of these $n$ probabilities is the number of novel entries expected by chance, and to express it as a percentage, this sum was divided by $n$ and the product was multiplied by 100. The resulting estimates of chance performance for each value of $n$ are shown in Table 2 .

Figure 1 reveals that the percentage of novel entries in the first $n$ opportunities fell from $85.3 \%$ to $78.0 \%$ when the number of arms was increased from 8 to 16 and re- mained at about that level until the 48 -arm condition, by which condition performance fell to $70.1 \%$. A one-way analysis of variance of phase means revealed a significant effect of conditions $[F(5,20)=5.14, p<.05]$, and planned comparisons of adjacent phase means revealed that performance in the 16-arm condition was significantly lower than that in the 8 -arm condition $[t(4)=4.17, p<$ $.05]$ and that performance in the 48-arm condition was significantly lower than that in the 40 -arm condition $[t(4)=3.18, p<.05]$. None of the other planned comparisons involving adjacent means was significant.

The phase mean for each number-of-arms condition was also compared with chance performance, using singlesample $t$ tests. The percentage of novel entries in the first $n$ opportunities remained significantly greater than chance until all 48 arms had been added, at which point it failed to exceed chance $\left[t_{8}(4)=5.51, p<.05 ; t_{16}(4)=\right.$ $3.25, p<.05 ; t_{24}(4)=2.51, p<.05 ; t_{32}(4)=3.59, p<.05$; $\left.t_{40}(4)=2.71, p<.05 ; t_{48}(4)=1.53, p>.05\right]$.

The probability of being correct on successive choices provides an additional analysis of performance on radial mazes. Olton and Samuelson (1976) described a statistic that depicts the observed probability of a correct response on successive choices within a trial after it has been corrected for chance selection. The details of how this statistic is calculated may be found in the Appendix. The statistic yields a value of zero when performance is at chance levels, a positive value when performance exceeds chance, and a negative value when performance is below chance. Moreover, the size of the statistic reflects

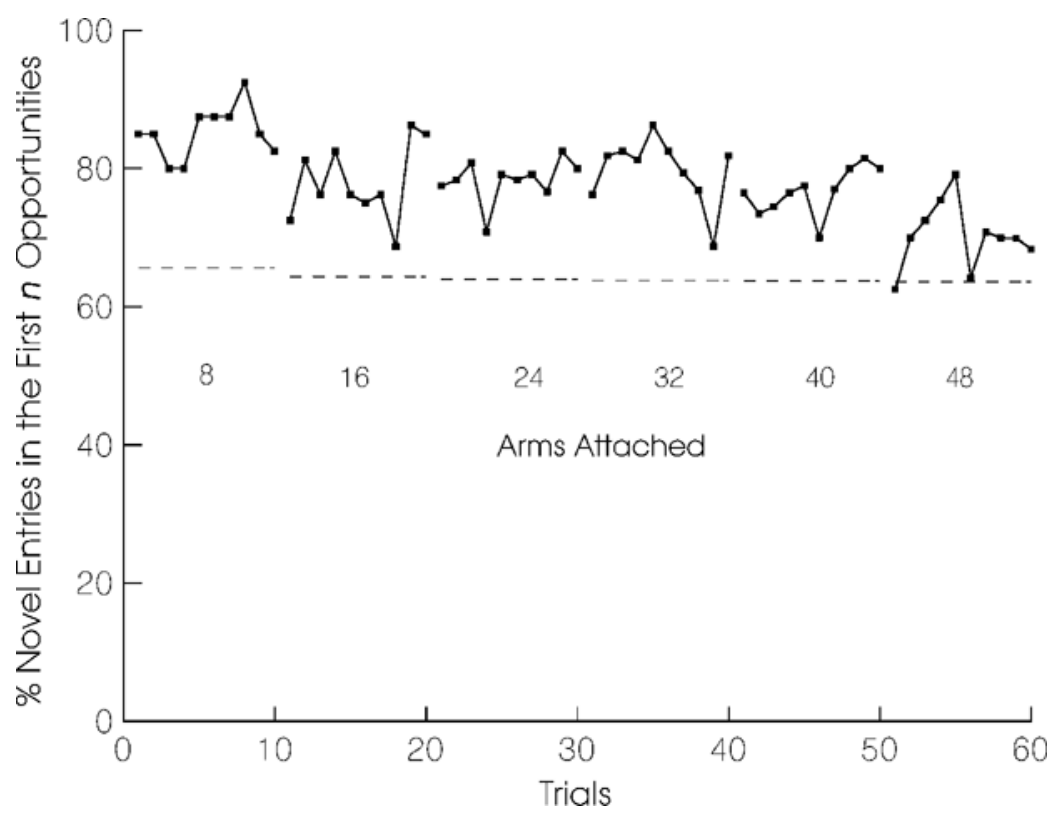

Figure 1. Percentage of novel entries in the first $n$ opportunities with 8, 16, 24, 32, 40, and 48 arms attached to random locations in Experiment 1. Chance performance in each phase is shown as a dotted line. 
Table 2

Estimates of Chance Performance in Experiment 1 Based on Random Sampling With Replacement

\begin{tabular}{rc}
\hline$n$ & \% Correct \\
\hline 8 & 65.64 \\
16 & 64.39 \\
24 & 63.99 \\
32 & 63.79 \\
40 & 63.68 \\
48 & 63.60 \\
\hline
\end{tabular}

the degree to which the performance is greater than or less than chance: perfectly correct performance $=+100.0$, whereas perfectly incorrect performance $=-100.0$.

Figure 2 shows the probability of being correct on successive choices in each condition. Note that the first choice is not shown, since the probability of being correct on the first choice is necessarily 1.0. In all cases, the data show a general decline in performance as the choices progressed. Except in the case of the 8 -arm condition, performance appears to have declined to chance levels as the number of choices approached $n$. This tendency toward chance responding was especially prevalent in the 48-arm condition, consistent with the failure of the 48-arm condition to produce a percentage of novel choices in the first $n$ opportunities that was significantly greater than the estimate of chance performance.

\section{Error Clusters}

In the eight-arm condition, two of the randomly selected arm locations turned out to be immediately side by side-henceforth referred to as the doublet. Totaled over all 5 rats, during the 10 trials of Phase 1, there were 59 reentries during the first eight choices. Of these, 30 were to the doublet and 29 to the other six arms. Thus, $51 \%$ of the errors were directed to the doublet during this phase. A chi-square analysis revealed this to be a significantly greater percentage of errors than would be expected by chance $\left[\chi^{2}(1)=21.0, p<.05\right]$. There were 50 occasions (5 rats over 10 trials) during Phase 1 in which one arm of the doublet was entered for the first time during the first eight choices. It is interesting to note that on $25(50 \%)$ of these occasions, the next response was to the other arm of the doublet. In the 16-arm condition, the random arm locations selected resulted in an additional doublet, as well as a triplet, of arms. Totaled over all 5 rats, of 138 total reentry errors during the first eight choices over the 10 trials of Phase 2, 37 were made to the triplet, 50 to the original doublet, and 26 to the new doublet, leaving only 25 errors directed to the remaining nine arms. Thus, $27 \%$ of the reentry errors were to the triplet, $36 \%$ to the original doublet, and $19 \%$ to the new doublet. Chi square analyses revealed that these deviations from expectancy were all significant: $\left[\chi^{2}\right.$ Doublet 1 $(1)=71.1, p<.05 ; \chi^{2}$ Doublet $2(1)=5.2, p<.05 ; \chi^{2}$ Triplet $(1)=5.9, p<.05]$. Again, it is interesting to note that of the 50 occasions on which one arm of each of the two doublets was entered for the first time during the first 16 entries, on $29(58 \%)$ and 23 (46\%) of these occasions, respectively, the next response was to the other arm of that doublet.

\section{Satiation Test}

The mean time taken to eat the first 24 pellets $(148.2 \mathrm{sec})$ was not significantly shorter $[t(4)=0.52, p>$ $.05]$ than that taken to eat the last 24 pellets $(159.3 \mathrm{sec})$.

\section{Discussion}

The data from Experiment 1 revealed the presence of a confound between number of arms and the spatial locations of those arms. The percentage of novel entry data are not systematic and suggest that the spatial arrangement of arms may have been more influential than the number of arms. By chance, the 16-arm condition contained two pairs of doublets and a triplet that, among them, attracted a lot of errors, and this accounted, at least in part, for the decline in performance when 8 arms were increased to 16. Moreover, the addition of the final set of 8 arms did more than add eight new locations. The increase from 40 to 48 arms also completed the maze, eliminating the last gaps between adjacent arms and making the maze a complete circle. It may have been this change in configuration that resulted in a further reduction in performance as the final 8 arms were added. The finding that the doublets and the triplet attracted more than their fair share of errors is consistent with the hypothesis that closely spaced arms are difficult to distinguish, albeit attractive, the latter presumably because they represent dense food patches.

In some ways, the data from Experiment 1 accord well with those reported by Olton and Samuelson (1976). Overall, performance was good, and the probability of being correct on successive choices showed a steady decline during the course of a trial in all the conditions. The latter finding suggests that the decline resulted from a failure of memory due to retroactive interference as more and more choices were made. On the other hand, contrary to the findings of Olton et al. (1977) and Roberts (1979), the results showed a significant decline in percentage of novel entries in the first $n$ opportunities as 8 arms were increased to 16 . However, the variations in spatial separation among the arms in the present research make this finding difficult to interpret. Finally, the satiation test data suggest that the decline in performance in the final phase, when all 48 arms had been added, was not due to satiation. The rat did not slow down significantly when eating the last 24 of 48 pellets. The question of whether number of food locations or spatial arrangement of food locations is more important in determining performance on the radial maze was further explored in Experiment 2.

\section{EXPERIMENT 2}

Experiment 1 suggested that the spatial separation between adjacent arms might have been a more potent de- 

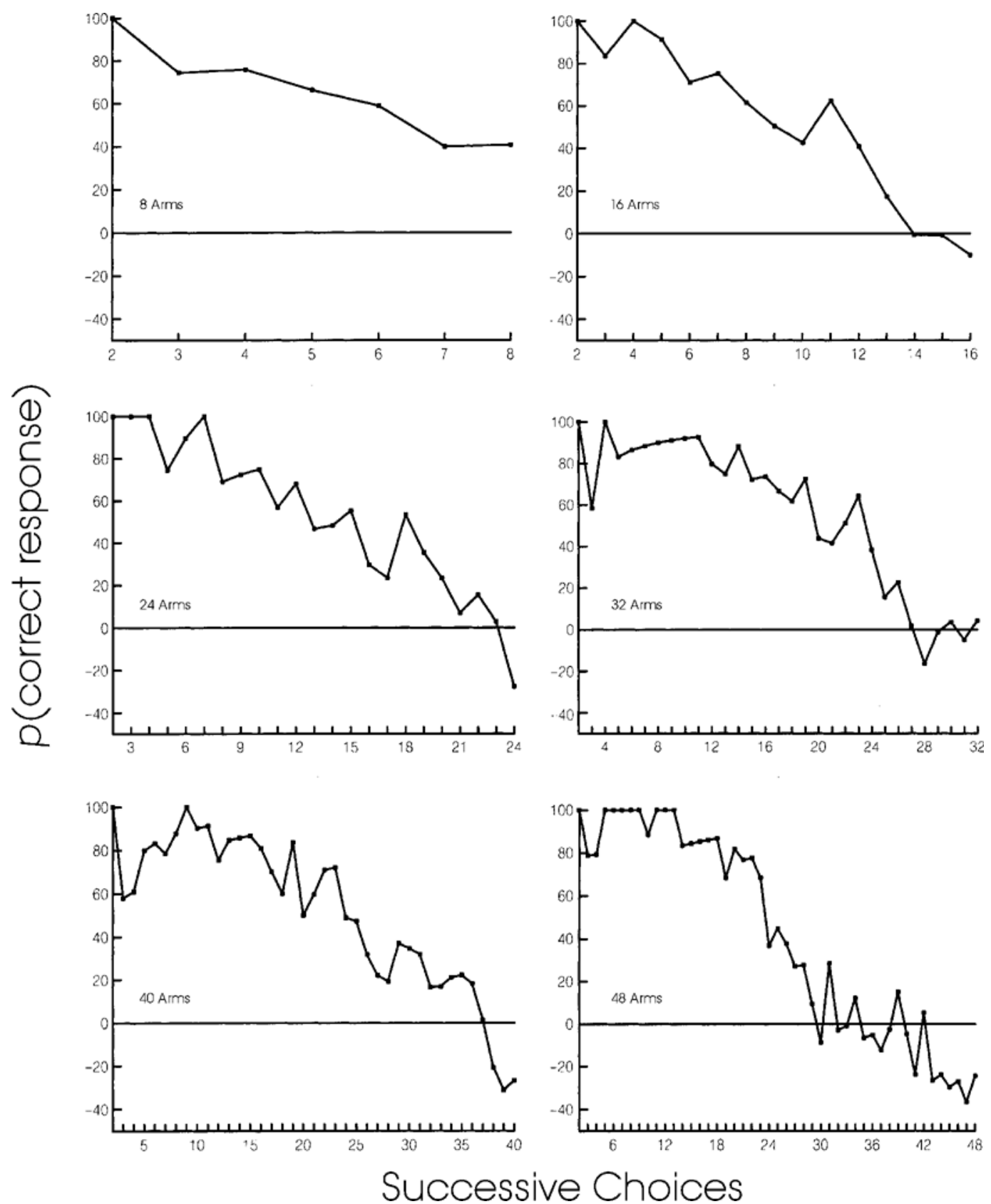

Figure 2. Probability of a correct response on successive choices with $8,16,24,32,40$, and 48 arms added in random locations in Experiment 1 . The first choice is not shown, since it is necessarily correct. The horizontal line at 0 represents chance performance.

terminant of performance than the number of arms on the maze. Experiment 2 was designed to assess systematically the relative contributions of the number of arms attached to the maze and the spatial separation among those arms as hungry rats foraged for food. In successive phases, $8,16,24$, and 48 arms were attached. Within each phase, in alternating sessions, arms were attached to the maze that were spread as far apart or clustered nearly as tightly together as the circumference of the central platform would permit.

\section{Method}

\section{Subjects}

Five male hooded rats of the Long-Evans strain, obtained from Charles River in Montreal and with prior experience both in operant chambers and on a small eight-arm radial maze, were used. The 
housing and feeding arrangements were the same as those in Experiment 1 .

\section{Apparatus}

The same apparatus and setting as those in Experiment 1 were employed in the present experiment.

\section{Procedure}

Preliminary training. With eight arms attached, the rats were given 10 trials of preliminary training at the rate of 2 trials per day in order to familiarize them with the maze. During these preliminary sessions, the arms were attached tightly together in Locations 1-8 in the morning session and spread as far apart as possible in Locations $1,7,13,19,25,31,37$, and 43 in the afternoon session. Thus, the rats were exposed to 4 trials in each of the two eight-arm configurations.

Training proper. In four successive phases, each consisting of 20 trials, $8,16,24$, or 48 arms were attached to the maze. There were two sessions per day, and in one of these sessions the arms were attached to the maze as far apart as possible, whereas in the other daily session they were clustered together almost as tightly as the central platform would permit with $0.5 \mathrm{~cm}$ between the edges of adjacent arms where they joined the platform. For the first 5 days in each phase, the rats were run in the order tight-spread-out, whereas during the second 5 days they were tested in the order spread-out-tight. In the final phase, there was no distinction between tight and spread-out, and so there were only 10 trials in this phase. The exact locations of the arms in both conditions in the first and second halves of each phase are shown in Table 3. The maze washing, trial-ending criteria, and data recording were all the same as those in Experiment 1.

\section{Memory for Locations}

The percentage of novel entries in the first $n$ opportunities was recorded over the course of Experiment 2. These data, together with estimates of chance performance, are shown in Figure 3.

The data reveal that in the spread-out configurations, the percentage of novel entries in the first $n$ opportunities fell from $96.0 \%$ to $83.0 \%$ and then to $71.9 \%$ as the number of arms was increased from 8 to 16 and then to 24 . Performance fell only slightly more, to $67.3 \%$, when all 48 arms were added. In the tight configurations, on the other hand, performance changed little as arms were added, declining from a relatively poor $75.5 \%$ novel entries in the first $n$ opportunities to $74.3 \%$ and then to $71.5 \%$ as the number of arms was increased from 8 to 16 and then to 24 .

A configuration $\times$ number-of-arms repeated measures analysis with two levels of configuration (tight vs. spread-out) and three levels of number of arms $(8,16$, or
24) was carried out on the phase means. This analysis revealed significant main effects of both configuration $[F(1,4)=17.8, p<.01]$ and number of arms $[F(2,8)=$ $23.8, p=.001]$, as well as a significant configuration $\times$ number-of-arms interaction $[F(2,8)=25.7, p<.001]$.

Planned comparisons revealed that there was a significant difference between the tight and the spread-out configurations in the 8 -arm $[t(4)=8.08, p<.05]$ and the 16 -arm $[t(4)=2.13, p<.05]$ configurations, but not in the 24-arm configurations [ $t(4)=0.49, p>.05]$. Planned comparisons also showed that in the tight configurations, there were no significant differences among any of the adjacent phase means for 8, 16, or 24 arms. Finally, planned comparisons further revealed that in the spreadout configurations, performance was significantly better in the 8 -arm condition than in the 16 -arm condition $[t(4)=4.46, p<.05]$ but that performance in the 16 -arm condition was not significantly better than that in the 24 -arm condition $[t(4)=1.0, p>.05]$.

In addition, each of the phase means in the 8-, 16-, 24-, and 48-arm conditions was compared with chance performance, using single-sample $t$ tests. Performance was significantly better than chance in the tight $[t(4)=$ $5.1, p<.05]$ and the spread-out $[t(4)=23.5, p<.05]$ 8 -arm configurations, in the tight $[t(4)=4.0, p<.05]$ and the spread-out $[t(4)=5.5, p<.05] 16$-arm configurations, and in the tight $[t(4)=3.9, p<.05]$ and the spread-out $[t(4)=4.1, p<.05] 24$-arm configurations. However, performance in the 48-arm configuration was not significantly better than chance $[t(4)=0.6, p>.05]$.

Figure 4 shows the probability of being correct on successive choices (Olton \& Samuelson, 1976) in the 8-arm, the 16-arm, the 24-arm, and the 48-arm conditions in both the tight and the spread-out configurations. Although the curve is essentially flat in the case of the 8-arm spread-out condition, the data otherwise show a general decline in performance over successive choices. As in Experiment 1, except in the 8-arm spread-out condition, performance approached chance levels toward the end of a trial. Once again, as in the previous experiment, this decline to chance levels was especially evident in the 48-arm condition, in which the number of arms was maximal and the separation among them was minimal.

\section{Patterning in Responding}

Although there are many ways to assess the presence of patterns in responding on a radial maze, Olton et al.

Table 3

Arm Locations in Each of the Four Successive Phases in Experiment 2

\begin{tabular}{cclrrrrrrrrrrrc}
\hline Phase & Arms & Configuration & \multicolumn{1}{c}{ A } & \multicolumn{1}{c}{ Sessions } & \multicolumn{1}{c}{ Arm Locations } \\
\hline 1 & 8 & tight & 1 & 3 & 5 & 7 & 9 & 12 & 14 & 16 & 18 & 20 & 1 to 8 \\
& 8 & spread out & 2 & 4 & 6 & 8 & 10 & 11 & 13 & 15 & 17 & 19 & every 6th location \\
2 & 16 & tight & 21 & 23 & 25 & 27 & 29 & 32 & 34 & 36 & 38 & 40 & 1 to 16 \\
& 16 & spread out & 22 & 24 & 26 & 28 & 30 & 31 & 33 & 35 & 37 & 39 & every 3rd location \\
3 & 24 & tight & 41 & 43 & 45 & 47 & 49 & 52 & 54 & 56 & 58 & 60 & 1 to 16 \\
& 24 & spread out & 42 & 44 & 46 & 48 & 50 & 51 & 53 & 55 & 57 & 59 & every 2nd location \\
4 & 24 & both & 61 & 62 & 63 & 64 & 65 & 66 & 67 & 68 & 69 & 70 & 1 to 48 \\
\hline
\end{tabular}




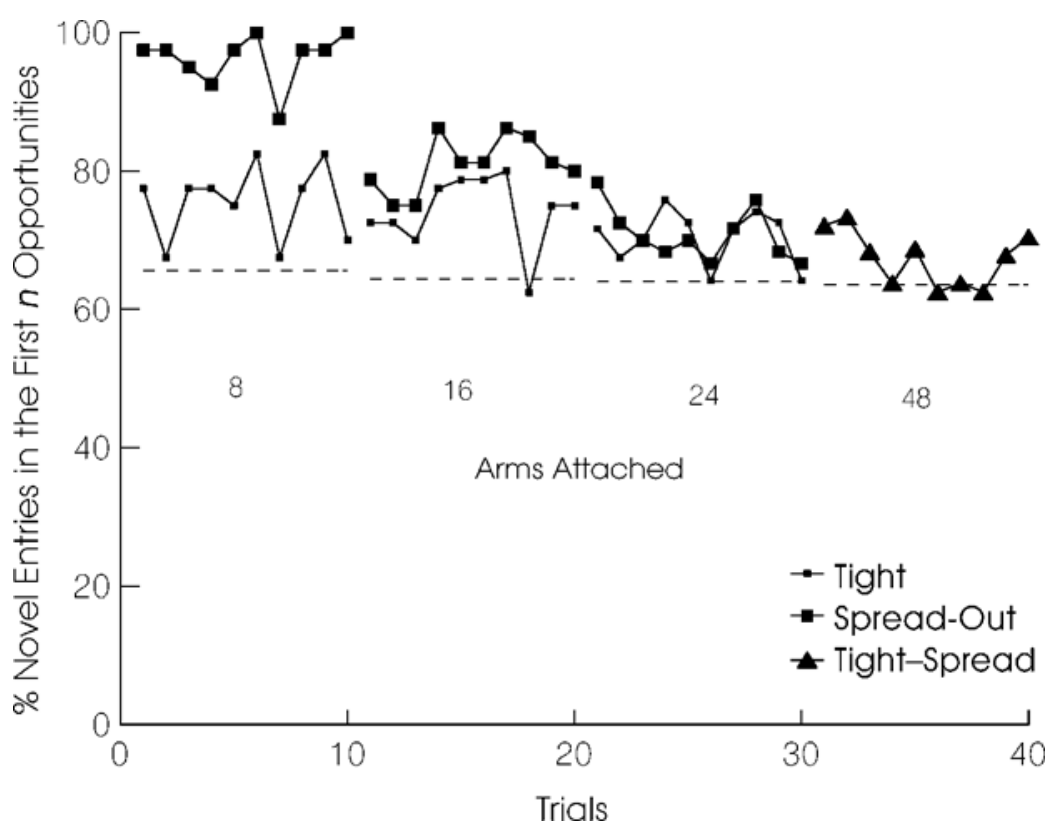

Figure 3. Percentage of novel entries in the first $n$ opportunities with 8, 16, 24, and 48 arms added in spread-out and tight configurations in Experiment 2. Note that in the case of 48 arms, spread-out and tight are one and the same thing. Chance performance in each phase is shown as a dotted line.

(1977) recorded the number of arms separating a chosen arm from the just previously chosen arm. Positive numbers were used to indicate a clockwise movement, and negative numbers were used to represent a counterclockwise movement. For example, if the rat went clockwise and skipped two arms, going from Arm 1 to Arm 4, +3 was recorded. Figure 5 shows the frequency distributions of such choices, represented as percentages to facilitate comparisons among the different conditions. Completely random choices would result in a flat distribution, but as Figure 5 shows, the distributions are far from flat. Instead, they tend to have two peaks, one at +1 or +2 and the other at -1 or -2 . These distributions suggest that the rats tended to move around the maze in clockwise or counterclockwise directions, selecting adjacent arms or skipping every other arm in their circuits. This tendency was more pronounced in the spread-out than in the tight configurations, especially in the 8-arm conditions. Fully $95.1 \%$ of choices in the 8 -arm spreadout condition were either +1 or -1 . The total of such choices in the 16-arm spread-out, 24-arm spread-out, and 48 -arm configurations were $46.5 \%, 15.2 \%$, and $22.1 \%$, respectively. Especially with 24 and 48 arms, the rats tended to skip every other arm in their circular journeys, presumably because, in these conditions, the arms were closer together.

\section{Discussion}

The results of Experiment 2 amplify and extend the results of Experiment 1 and confirm that the changes in performance seen in that experiment were due primarily to decreases in the separation of adjacent arms, rather than to increases in the number of arms. There are a number of reasons why tightly clustered arms might lead to poor performance on a radial maze. First, tightly clustered arms might be confusing and difficult to distinguish (Staddon, 1983). Alternatively, entering adjacent tightly clustered arms requires tight turns that rats might be unwilling or unable to make (Yoerg \& Kamil, 1982). Finally, rats might perform best when they can distribute successive searches widely, a strategy prohibited by tightly clustered food locations (Grobety \& Schenk, 1992). However, regardless of the reason for which spatial separation among food locations exerts such an effect on performance, this factor must be controlled if the effect of number of food locations on spatial memory is to be explored further.

\section{EXPERIMENT 3}

Experiments 1 and 2 revealed that spatial separation among the arms on a radial maze was an important determinant of performance on the maze, with percentage of correct responses decreasing as the arms became more and more tightly grouped. With a constant-diameter central platform, the arms on a radial maze necessarily become more and more tightly clustered as arms are added. What is needed is a means of holding spatial separation between adjacent arms constant as the number of arms is systematically increased. However, controlling spatial 

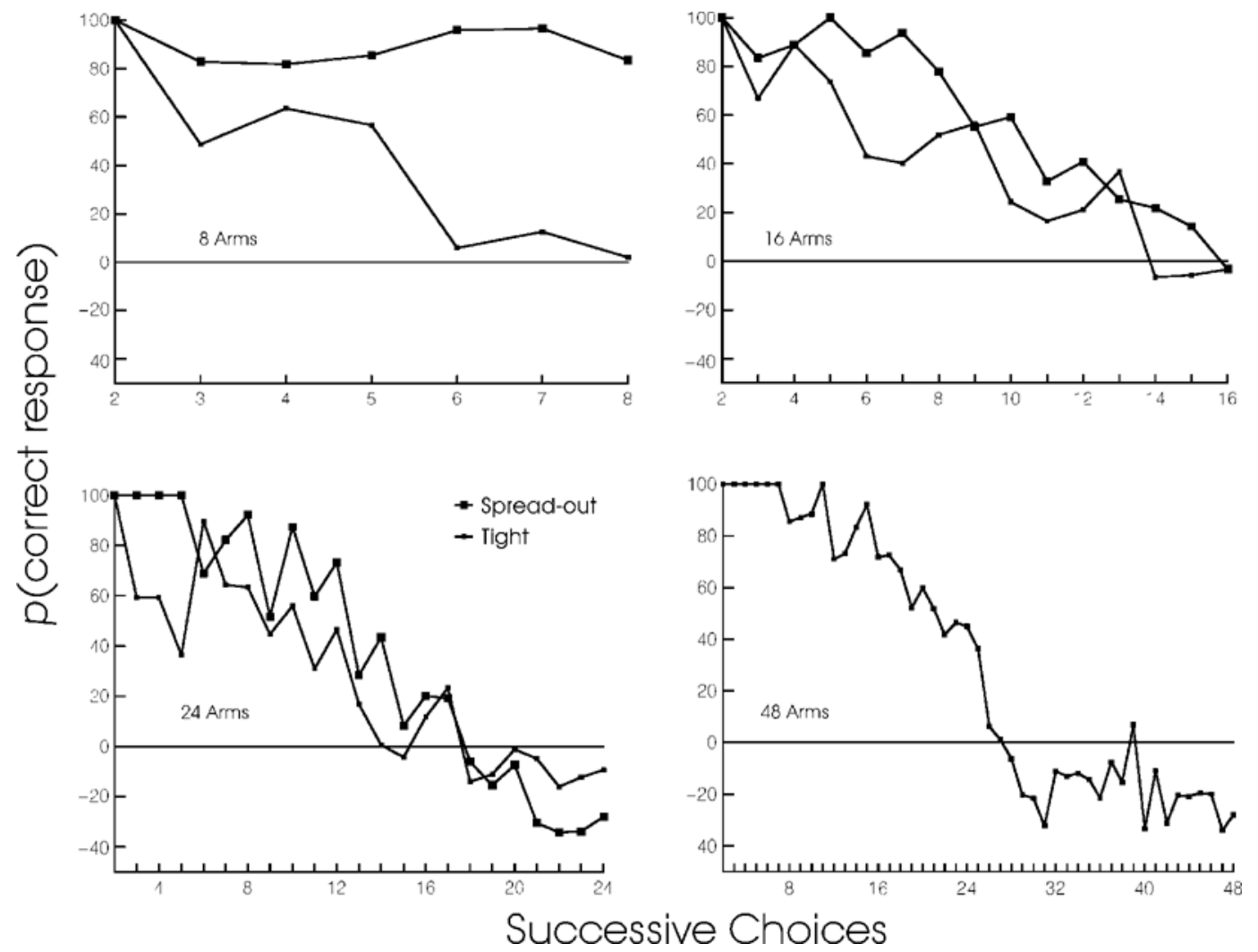

Figure 4. Probability of a correct response on successive choices with $8,16,24$, and 48 arms added in spreadout and tight configurations in Experiment 2. Note that in the case of 48 arms, spread-out and tight are one and the same thing. The first choice is not shown, since it is necessarily correct. The horizontal line at 0 represents chance performance.

separation among arms on a radial maze as arms are added would require an increase in the diameter of the central platform. In the case of the maze used in Experiment 2, the separation between the midlines of the adjacent arms where they joined the central platform was about $60 \mathrm{~cm}$, with 8 arms spaced out as far as possible, and to maintain that separation with 48 arms would require a central platform over $9 \mathrm{~m}$ in diameter. Accordingly, we decided to abandon the radial maze altogether and to use an apparatus similar to one designed by Brown and Terrinoni (1996). These investigators set vertical poles, each a $15.5-\mathrm{cm}$-tall dowel, into the plywood floor of a test box. The top of each pole contained a shallow well into which a food pellet could be placed. In order to check whether the poles were baited, rats had to rear up against them, thus making their choices easy to observe and record. We used freestanding food towers that were easy to move around, instead of dowels, and in successive phases, there were $8,16,24,32,40,48,16$, or 8 such towers arranged in a circle on the floor of the test room. As towers were added or removed, the diameter of the circle was increased or decreased, so that a constant $33 \mathrm{~cm}$ was maintained between the centers of adjacent towers. Each tower circle was itself surrounded by a circular wall slightly larger in diameter than the circle of towers, in order to ensure that the towers in each condition were equally distant from the perimeter of the research space. As rats tend to be thigmotaxic, such a control was deemed important.

\section{Method}

\section{Participants}

Six male hooded rats of the Long-Evans strain, obtained from Charles River in Montreal and with prior experience both in operant chambers and on a small eight-arm radial maze, were used. The housing and feeding arrangements were the same as those in Experiments 1 and 2 .

\section{Apparatus}

The 48 food towers used in the present experiment were constructed from cedar, and each was $9.0 \times 9.0 \times 15 \mathrm{~cm}$ high. The towers were coated with a clear Varathane varnish, and each had a food cup mounted in the center of the top surface. The food cups were the same as those used in the first two experiments. The towers designated for use in each of the eight successive phases in the experiment were set in a circle on the floor in the center of the test room so that the distance between the centers of the food cups on adjacent towers was $33 \mathrm{~cm}$. Surrounding this circle of towers was a 


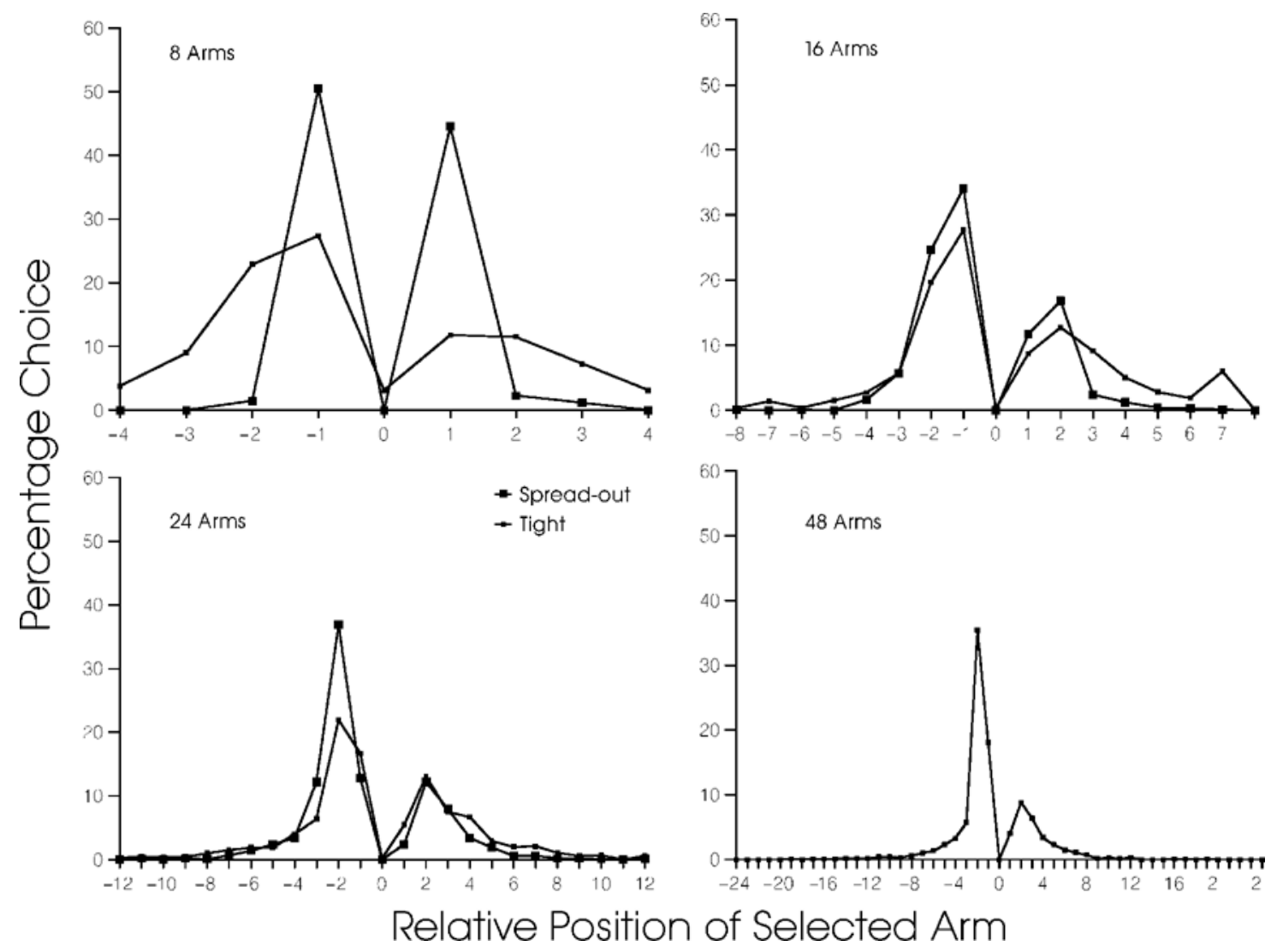

Figure 5. Frequency distributions of the number of arms for which choices are removed from the previous choice, with $8,16,24$, and 48 arms added in spread-out and tight configurations in Experiment 2 . Note that in the case of 48 arms, spread-out and tight are one and the same thing. Negative numbers refer to counterclock wise shifts, whereas positive numbers refer to clockwise shifts.

circular arena wall constructed of Masonite panels. Each section of this wall was $244 \mathrm{~cm}$ long $\times 40 \mathrm{~cm}$ high $\times 0.5 \mathrm{~cm}$ thick and was finished on one side with a white impervious coating. Sufficient overlapping wall sections were fastened together by carriage bolts, so that the resulting circular wall was $40 \mathrm{~cm}$ greater in diameter than the outside perimeter of the circle of towers. This allowed the rats to move freely on the inside or the outside of the tower ring. The inner surface of the wall was white, again to provide a backdrop against which it would be easier to observe the rats. The test room itself was $5.6 \times 6.0 \mathrm{~m}$ and was just large enough to contain a circle of 48 towers spaced $33 \mathrm{~cm}$ apart that was, in turn, surrounded by the arena wall. There was a door and a blackboard on the west wall, two large one-way mirror windows on the south wall, and a large window on the north wall. Tower locations were numbered clockwise, starting with the location that was due east. A $3.0 \times 3.0 \mathrm{~cm}$ magentacolored Post-it note was attached to the arena wall above the towers in the northeast, southeast, southwest, and northwest locations, and a $3.0 \times 3.0 \mathrm{~cm}$ yellow-colored Post-it note was attached to the arena wall in the north, east, south, and west locations. The purpose of these markers was to assist the researchers in accurately recording tower choices, so the marker was attached in such a way that it was visible to the experimenter when data were recorded. As was the case in the first two experiments, these markers were also visible to the rats, but once again the researchers could detect no evidence

Table 4

Tower Configurations in the Successive Phases of Experiment 3

\begin{tabular}{cccc}
\hline Phase & Number of Towers & Diameter of Towers $(\mathrm{cm})$ & Diameter of Wall $(\mathrm{cm})$ \\
\hline 1 & 8 & 84 & 124 \\
2 & 16 & 168 & 208 \\
3 & 24 & 252 & 292 \\
4 & 32 & 336 & 376 \\
5 & 40 & 420 & 460 \\
6 & 48 & 504 & 544 \\
7 & 16 & 168 & 208 \\
8 & 8 & 84 & 124 \\
\hline
\end{tabular}


that the rats made use of them in any way. The carpeted floor of the room was completely covered with a 1.6-mm-thick vapor barrier to facilitate between-trials washing of the apparatus and to ensure the health of the rats. The food cups were baited with 45-mg Noyes pellets. The observer sat in a chair located on top of a 76-cm-high table located in the northwest corner of the room, about $60 \mathrm{~cm}$ beyond the arena wall.

Procedure

There were eight successive phases involving $8,16,24,32,40$, 48,16 , and 8 towers. The diameters of the tower circles and the surrounding arena walls are shown in Table 4.

Preliminary training. With the eight-tower circular arena wall set in the center of the room, the rats were given preliminary training trials, initially using three training towers. One of these was $9.0 \mathrm{~cm}$ high, the second was $12.0 \mathrm{~cm}$ high, and the third was a full $15.0 \mathrm{~cm}$ high. After the rats had learned to look for food on the training towers, a full circle of eight regular towers was set out within the eight-tower wall. Training on these towers continued for 10 sessions at the rate of two trials per day. By the end of preliminary training, all the rats regularly visited all the towers and ate the food in each food cup on a given trial.

Training proper. Following preliminary training, the rats were given two trials per day, with the first daily trial session starting at about $10 \mathrm{a} . \mathrm{m}$. and the second beginning at about $2 \mathrm{p} . \mathrm{m}$. At the start of each trial, the floor area within $40 \mathrm{~cm}$ of the towers was washed with a mild soap solution, and all towers were baited with one 45-mg Noyes pellet. The rat was placed within the arena at a randomly selected northwest, northeast, southwest, or southeast point close to the arena wall. The researchers did not place the rat in the center of the arena at the start of each trial because, as the diameter of the circle of towers was increased during the course of the experiment, it would have become necessary to enter the arena to do so. After being removed from the arena after the afternoon session, the rat was weighed and given a ration of lab chow sufficient to maintain its target weight. Beginning with a circle of 8 towers, after each set of 10 trials, an additional 8 towers were added to the maze, and the size of the arena expanded accordingly until, during the sixth phase, the circle consisted of 48 towers. During Phases 7 and 8 , the number of towers was reduced to 16 and 8 , respectively.

Trial-ending criteria. With $n$ referring to the number of towers in place, the rat remained in the arena on any trial until it met any one of the following criteria: (1) $n$ choices had been made, (2) $n$ minutes had passed, or (3) the rat attempted to climb out of the arena.

Data recording. After placing the rat in the arena, the researcher retreated to the observer's chair. Facsimiles of the maze were used to record the order of arm entries and related data.

\section{Results}

\section{Memory for Locations}

The percentage of novel visits in the first $n$ opportunities was recorded over the course of Experiment 3 . These data are shown in Figure 6 and reveal that the percentage of novel choices in the first $n$ opportunities fell from $79.9 \%$ in the 8 -tower condition to $75.9 \%$ in the 16 -tower condition, declined again to $71.2 \%$ in the 24 -tower condition, but then did not change much after that as the number of towers was increased to 32,40 , and finally to 48 .

The data also show that performance recovered to $76.0 \%$ and then to $80.0 \%$ when the number of towers was decreased, first from 48 to 16 and then from 16 to 8 . A one-way analysis of variance of phase means revealed a significant effect of conditions $[F(7,35)=3.29, p<.05]$, and planned comparisons of adjacent phase means revealed that performance in the 16-tower condition was significantly lower than that in the 8-tower condition $[t(5)=2.23, p<.05]$ and that, in the reversal, performance in the 16-tower condition was significantly poorer than

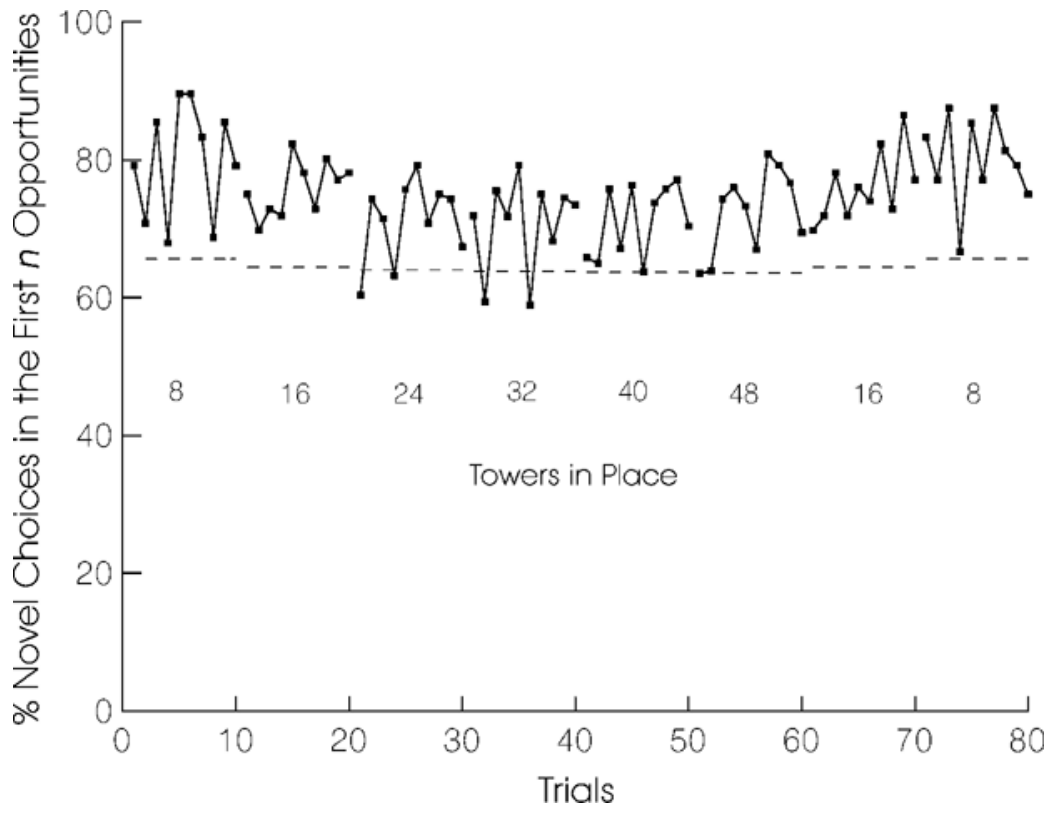

Figure 6. Percentage of novel choices in the first $n$ opportunities with 8, 16, 24, 32, 40, 48, 16, and 8 towers spaced $33 \mathrm{~cm}$ apart in Experiment 3. Chance performance in each phase is shown as a dotted line. 

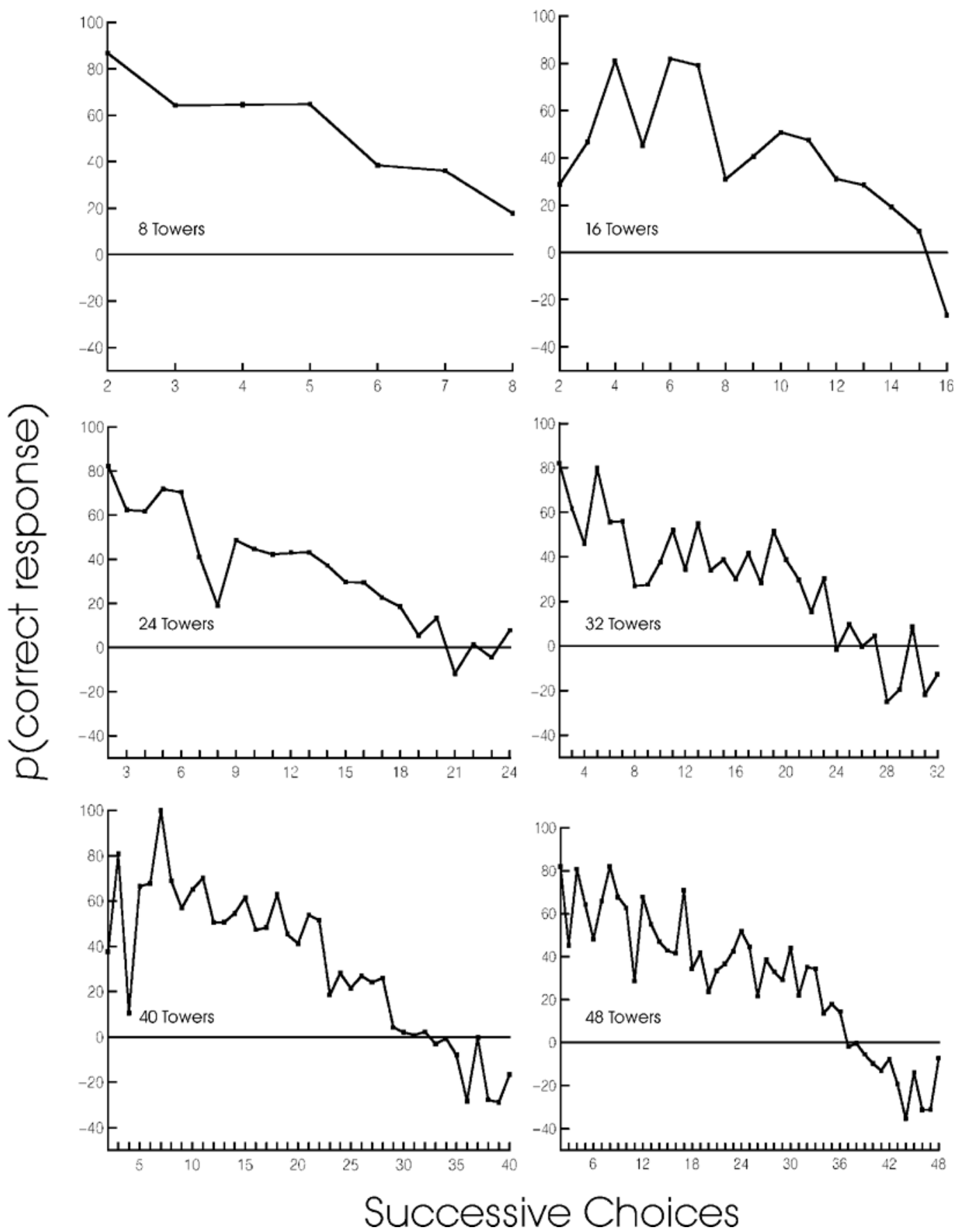

Figure 7. Probability of a correct response on successive choices with $8,16,24,32,40$, and 48 towers spaced $33 \mathrm{~cm}$ apart in Experiment 3. The first choice is not shown, since it is necessarily correct. The horizontal line at 0 represents chance performance.

that in the 8-tower condition $[t(5)=2.02, p<.05]$. None of the other planned comparisons involving adjacent means was significant.

In addition, each of the phase means in the 8-, 16-, 24-, 32-, 40-, and 48-tower, as well as in the 16- and 8 -tower reversal conditions, was compared with chance performance, using single-sample $t$ tests. Performance was significantly better than chance in the original 8 -tower $[t(5)=6.8, p<.05]$ and in the 16-tower $[t(5)=4.6, p<$ $.05]$ configurations, and also in the 16-tower $[t(5)=3.6$, $p<.05]$ and the 8 -tower $[t(5)=3.0, p<.05]$ reversal configurations. However, performance in the 24-tower $[t(5)=$ 


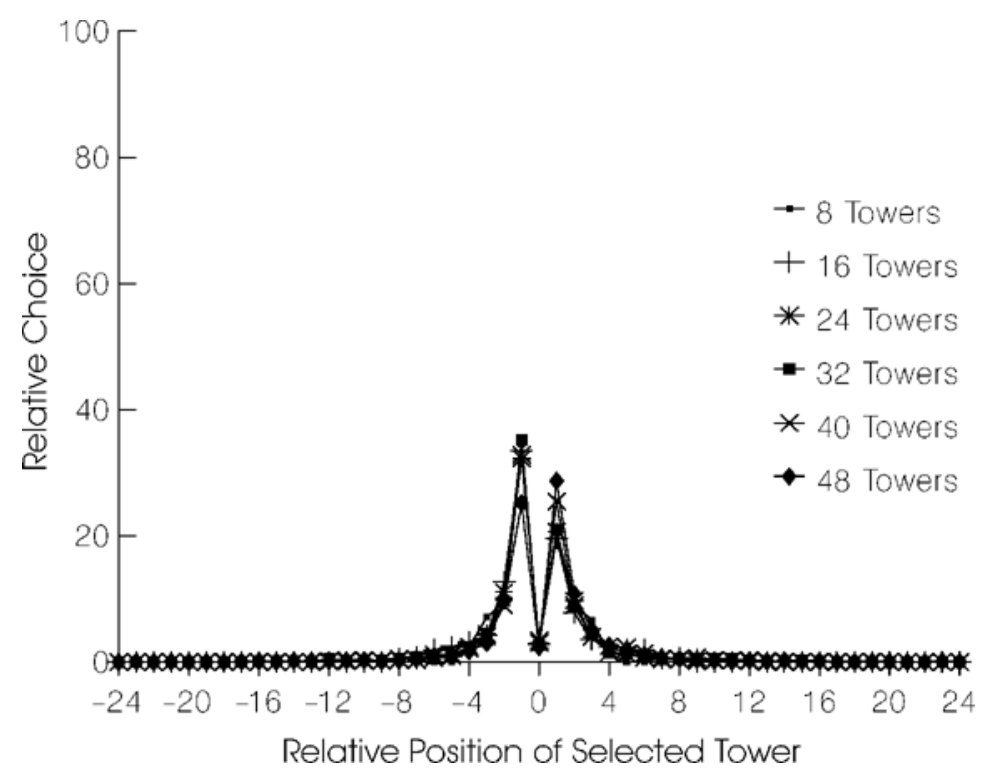

Figure 8. Frequency distributions of the number of towers for which choices are removed from the previous choice, with $8,16,24,32,40$, and 48 towers spaced $33 \mathrm{~cm}$ apart in Experiment 3. Negative numbers refer to counterclock wise shifts, whereas positive numbers refer to clockwise shifts.

1.6, $p>.05], 32$-tower $[t(5)=1.3, p>.05], 40$-tower $[t(5)=1.6, p>.05]$, and 48-tower $[t(5)=2.0, p>.05]$ configurations was not significantly better than chance.

Figure 7 shows the probability of being correct on successive choices over the first six phases. The data for Phases 7 and 8 were virtually identical to those shown in Phases 1 and 2 and are not shown. In all cases, the graphs reveal a general decline in performance over successive choices, and in all but the 8-tower condition, the curves intersect chance levels toward the end of the trial. In the 16-tower condition, performance was worse on Choices 2 and 3 than on Choice 4 , and in the 40 -tower condition, performance was worse on Choice 2 than on Choice 3. During these two phases, 2 of the 6 rats showed a strong tendency to direct their second choices and, sometimes, their third choices to the originally selected tower(s).

\section{Patterning in Responding}

The same technique as that used to assess patterning of choices in Experiment 2 was employed in Experiment 3 , with positive numbers indicating the degree of clockwise rotation and negative numbers indexing counterclockwise rotation. Figure 8 shows the frequency distributions of these choices over the first six phases. The data are represented as percentages to facilitate comparisons among the different conditions. All the distributions show marked peaking at +1 and -1 , regardless of the number of towers. Moreover, the data in Figure 8 suggest little difference in patterning as the number of towers was increased from 8 to 48 .

The percentage of total choices that were +1 or -1 in each phase ranged from $52.9 \%$ to $60.0 \%$, with a mean value of $55.8 \%$. A one-way repeated measures analysis of variance of the percentage of total choices that were +1 or -1 over the first six phases revealed no significant effect of number of towers $[F(5,25)=1.24, p>$ $.05]$.

\section{Discussion}

Because the spacing between adjacent towers was held constant at $33 \mathrm{~cm}$, it may be confidently asserted that the changes in performance as 8 towers were increased to 16 and as 16 towers were decreased to 8 were due to the number of food locations, not to the spacing among them, as had been the case, at least partially, in the first two experiments. Of course, this does not mean that spacing was not a factor, but only that it was not confounded with number of towers. On the other hand, a separation of $33 \mathrm{~cm}$ between adjacent towers could not be described as a wide gap. In Experiment 2, the separation between the centers of the arm entrances in the 8 -arm spread-out condition was $60 \mathrm{~cm}$, and the actual separation between adjacent food cups was even more than that. One indication that separation, although controlled, was still a factor was the relatively poor performance in the 8 -tower condition. At about $80 \%$, it was somewhat less than the approximately $87 \%$ correct performance reported by Olton and Samuelson (1976) and much less than the almost perfect performance that occurred in the 8-arm spread-out condition in Experiment 2 in the present research.

The probabilities of correct responses on successive choices data were similar to those seen in the previous two experiments, and the general decline is consistent 
with a memory interpretation of the data. In fact, with any number of towers greater than eight, the rats appeared to be choosing randomly by the end of the trial. Such behavior would be expected when retroactive interference made recall difficult or impossible.

The patterning data from Experiment 3 reveal a somewhat greater tendency to choose adjacent food locations than had been the case in the first two experiments, in which the radial maze had been used, presumably because in this open-field environment there were even fewer restrictions on making such choices. On the radial maze used in Experiments 1 and 2, even though the arms were short and the sidewalls did not extend fully to the central platform, the rat nevertheless had to retreat much of the way toward the central platform in order to select an alternate food location. With the tower maze arrangement, on the other hand, there was nothing to prevent the rat from simply moving directly from tower to tower. The important point, however, is that there was no evidence that the changes in correct choices as the number of towers was increased or decreased were due to patterning. On balance, the changes in performance seem most amenable to a memory explanation.

\section{EXPERIMENT 4}

The results of Experiment 3 showed that with the separation between adjacent food locations held constant, there was a decrease in performance when 8 food locations were increased to 16. However, although the distance between adjacent food locations was held constant in Experiment 3 , it might still have been exerting some influence over responding as the number of towers was manipulated. The best evidence for this was substandard performance in the eight-tower condition, relative to that seen on a typical radial maze. Experiment 4 was a partial replication of Experiment 3 , in which the distance between adjacent food locations was doubled from 33 to $66 \mathrm{~cm}$. Although doubling the separation between adjacent towers was not expected to eliminate completely the influence of spacing, it was anticipated that it would make it closer to that typically seen on the radial maze and, thus, provide data on the effect of number of food locations on recall of those locations that is less contaminated by spatial factors.

\section{Method}

Subjects

Five male hooded rats of the Long-Evans strain, obtained from Charles River in Montreal and with prior experience both in operant chambers and on a small eight-arm radial maze, were used. The housing and feeding conditions were the same as those in Experiments 1, 2 , and 3. However, due to a reversal of the usual light:dark cycle in the laboratory, the rats were tested during the dark cycle, not during the light cycle, as had been the case in the first three experiments.

\section{Apparatus}

The food towers used in the present experiment were the same as those used in Experiment 3. However, the number of towers used in each of four successive phases in the experiment were set in a circle on the floor of the test room so that the distance between the centers of the food cups on adjacent towers was $66 \mathrm{~cm}$. As had been the case in Experiment 3, the tower circle was surrounded by a circular Masonite wall, white side in, that was, once again, $40 \mathrm{~cm}$ high and $40 \mathrm{~cm}$ larger in diameter than the tower circle. Although the same test room $(5.6 \times 6.0 \mathrm{~m})$ as that in Experiment 3 was used for the first three phases of the present experiment, Phase 4, involving 32 towers, had to be carried out in a larger room. This larger room was $19.8 \times 14.2 \mathrm{~m}$ and had windows on the north, east, and west walls and several doors on the south wall. Since this room was not carpeted, no vapor barrier was used to cover the floor of the arena in Phase 4. Unfortunately, access to this large room could not be secured during the entire experiment.

\section{Procedure}

There were four successive phases involving 8, 16, 24, and 32 towers. The diameters of the tower circles and the surrounding arena walls were as shown in Table 5.

Preliminary training. Preliminary training was carried out exactly as in Experiment 3.

Training proper. Following preliminary training and beginning with the 8-tower circle, the rats were given two trials per day, with the first daily trial session starting at about 10 a.m. and the second beginning at about 2 p.m. Exactly the same procedures as those in Experiment 3 were used on each trial. After each set of 10 trials, an additional 8 towers were added to the arena. There was no reversal back to fewer towers after the 32-tower condition.

\section{Results}

\section{Memory for Locations}

The percentage of novel visits in the first $n$ opportunities was recorded over the course of the experiment. These data are shown in Figure 9 and show that the percentage of novel choices in the first $n$ opportunities fell from $86.8 \%$ in the 8 -tower condition to $79.6 \%$ in the 16 -tower condition and declined again to $72.7 \%$ in the 24-tower condition, but then decreased minimally as the number of towers was further increased to 32 .

A one-way analysis of variance of phase means revealed a significant effect of conditions $[F(3,12)=7.76$, $p<.05]$, and planned comparisons revealed that performance in the 16-tower condition was significantly lower than that in the 8 -tower condition $[t(4)=2.64, p<.05]$

Table 5

Tower Configurations in the Successive Phases of Experiment 4

\begin{tabular}{cccc}
\hline Phase & Number of Towers & Diameter of Towers $(\mathrm{cm})$ & Diameter of Wall $(\mathrm{cm})$ \\
\hline 1 & 8 & 168 & 208 \\
2 & 16 & 336 & 376 \\
3 & 24 & 504 & 544 \\
4 & 32 & 672 & 712 \\
\hline
\end{tabular}




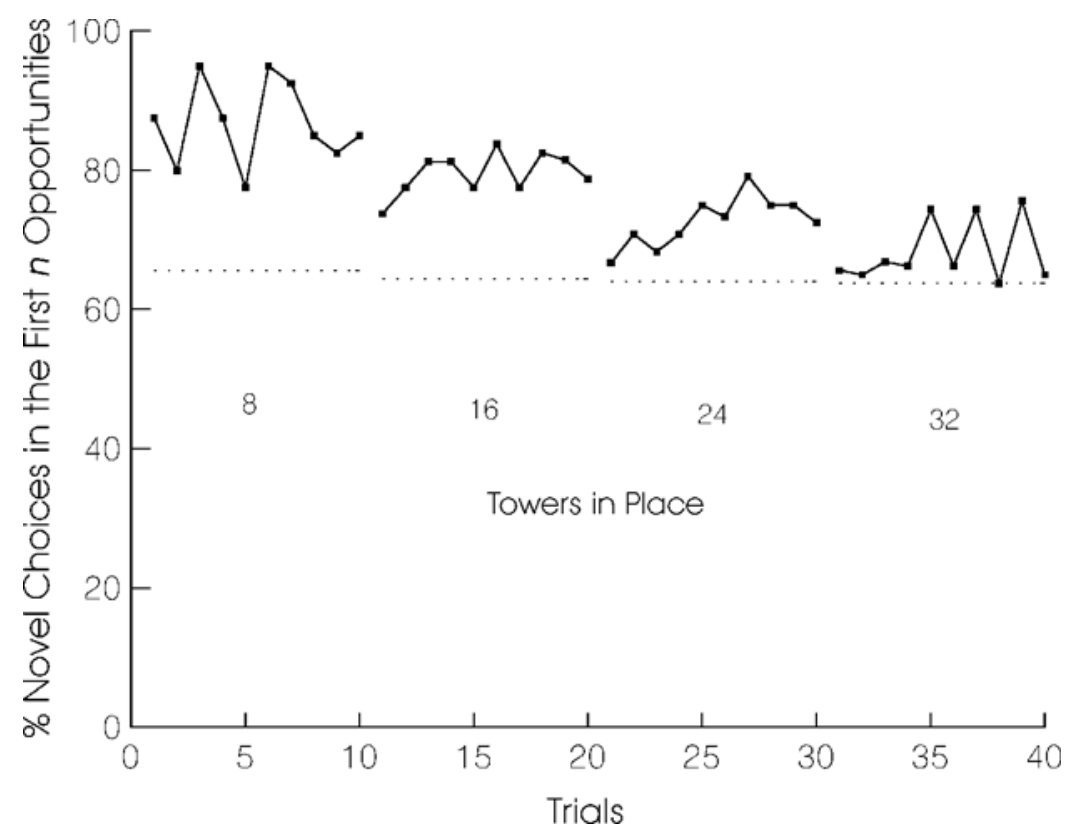

Figure 9. Percentage of novel choices in the first $n$ opportunities with $8,16,24$, and 32 towers spaced $66 \mathrm{~cm}$ apart in Experiment 4. Chance performance in each phase is shown as a dotted line.

and that performance in the 24-tower condition was significantly lower than that in the 16-tower condition $[t(4)=$ $3.55, p<.05$ ]. However, performance in the 32-tower condition was not significantly lower than that in the 24-tower condition $[t(4)=2.16, p<.05]$.

Each of the phase means in the 8-, 16-, 24-, and 32-tower conditions was also compared with chance performance, using single-sample $t$ tests. Performance was significantly better than chance in the 8-tower $[t(4)=7.6, p<$ $.05]$, the 16 -tower $[t(4)=8.0, p<.05]$, and the 24 -tower $[t(4)=3.5, p<.05]$ configurations, but not in the 32-tower $[t(4)=0.8, p>.05]$ configuration.

Figure 10 shows the probability of a correct selection on successive choices for all four phases. In all cases, the data show a general decline in performance as the trial progressed, although, in the 16-tower condition, performance on Choices 2-4 was worse than that on Choice 5, and in the 24-tower condition, it was worse on the second choice than on the third choice. Once again, this occurred because 2 of the rats tended to perseverate after their first choice before moving on. In all the phases, except possibly the 8-tower condition, performance approached chance as the number of choices in a trial progressed.

\section{Patterning in Responding}

The same technique as that used to assess patterning of choices in Experiment 3 was employed in Experiment 4, with positive numbers indicating the degree of clockwise rotation and negative numbers indexing counterclockwise rotation. Figure 11 shows the frequency distributions of these choices over all four phases. The data are once again represented as percentages to facilitate comparisons among the different conditions. All the distributions show marked peaking at +1 and -1 , as was the case in Experiment 3.

The percentage of total choices that were +1 or -1 in each phase ranged from $74.3 \%$ in the 8 -tower condition to $36.8 \%$ in the 32 -tower condition, with a mean value of $55.8 \%$ over all four conditions. A one-way repeated measures analysis of variance of the percentage of total choices that were +1 or -1 over the first six phases revealed a significant effect of number of towers $[F(3,12)=13.98$, $p<.05]$. Planned comparisons of adjacent phase means, however, revealed that only the difference between 24 and 32 towers was significant $[t(4)=4.36, p<.05]$.

\section{Discussion}

Once again, because the spacing between adjacent towers was held constant, this time at $66 \mathrm{~cm}$, it may be argued that the decreases in performance as 8 towers were increased to 16 and, again, as 16 towers were increased to 24 were due to number of food locations, not to the spacing among them. However, with intertower separation held constant at $66 \mathrm{~cm}$, the point at which performance declines leveled off was extended to 24 towers.

The probability of a correct response on successive choices data were similar to those seen in all the previous experiments in the present research, and once again, the general decline over choices is consistent with a memory interpretation of the data. The tendency for 

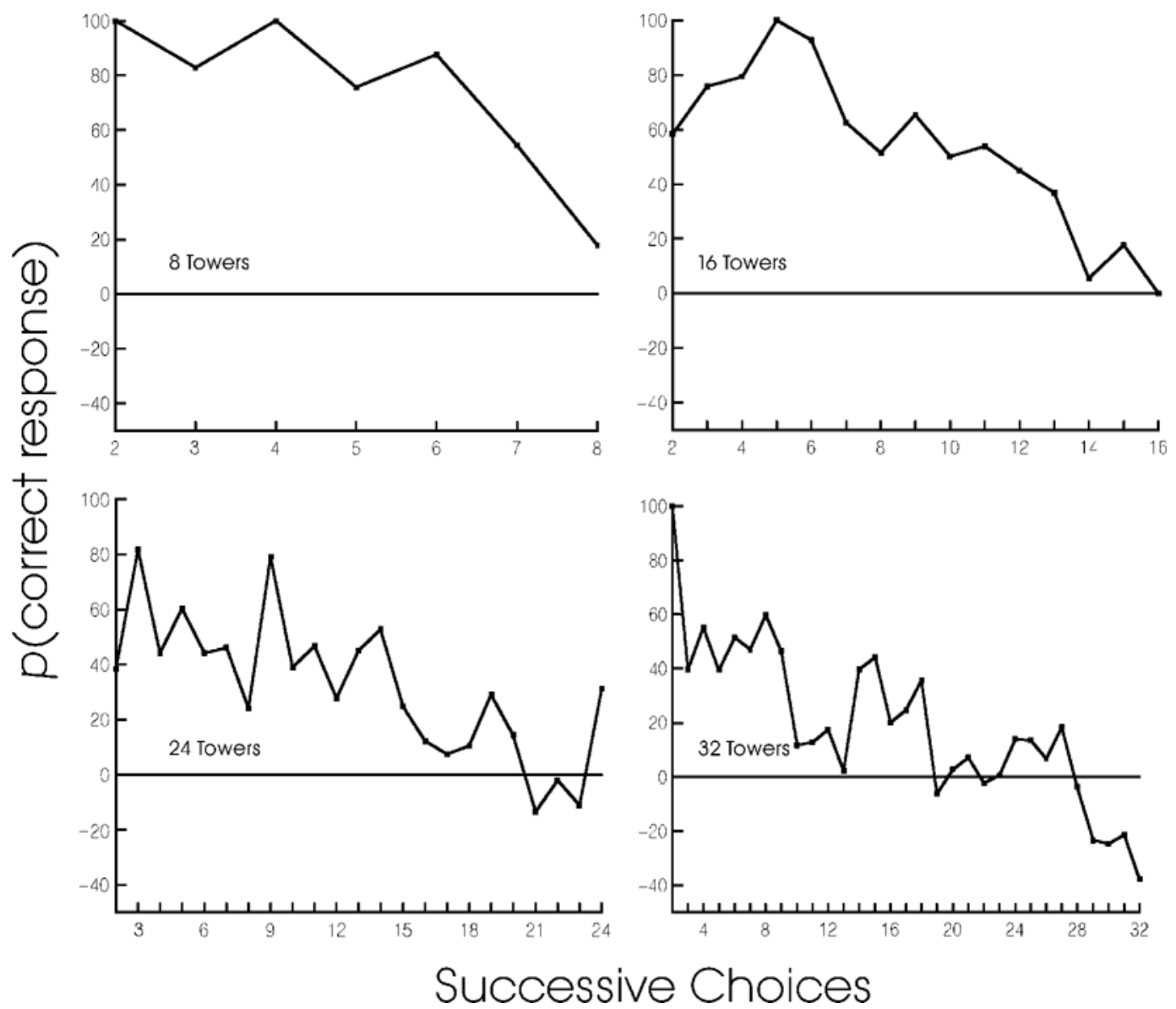

Figure 10. Probability of a correct response on successive choices with $8,16,24$, and 32 towers spaced $66 \mathrm{~cm}$ apart in Experiment 4 . The first choice is not shown, since it is necessarily correct. The horizontal line at 0 represents chance performance.

some rats to revisit their initial choice(s) before moving on in the 16- and 24-tower conditions replicates a similar tendency seen in Experiment 3. It is possible that a similar phenomenon occurred in Experiments 1 and 2 but was undetected. It is also possible that this tendency is peculiar to the tower arrangement. In any case, it was not seen in all the rats, and it remains to be seen whether it is an important phenomenon. The patterning data are also similar to those seen in Experiment 3 in suggesting a slightly greater tendency to choose adjacent food locations than had been the case on the spread-out radial maze configurations in Experiment 2. Once again, however, there is little evidence that the declines in correct choices as the number of towers was increased were correlated meaningfully with patterning. More specifically, the declines in the percentage of novel choices in going from 8 to 16 and from 16 to 24 locations were not accompanied by significant declines in the percentage of +1 and -1 rotations. Although there was a significant decrease in the tendency to select adjacent towers as 24 towers were increased to 32 towers, there was no corresponding decrease in correct choices. Once again, the changes in performance seem best attributed to failures of memory.

\section{GENERAL DISCUSSION}

In the present research, the results of the first two experiments pointed to the importance of the spatial arrangement of the arms on a radial maze. Experiment 1 revealed sporadic declines in the percentage of correct choices as sets of 8 arms were added to a radial maze in random locations. With the addition of the last set of arms, raising the total from 40 to 48 , performance not only declined, but also declined to chance. As was pointed out in the Discussion section for that experiment, there was reason to believe that this might be due more to the spatial arrangement of the arms than to the number of arms. Specifically, a pair of arms located immediately side by side in the 8 -arm condition and two 


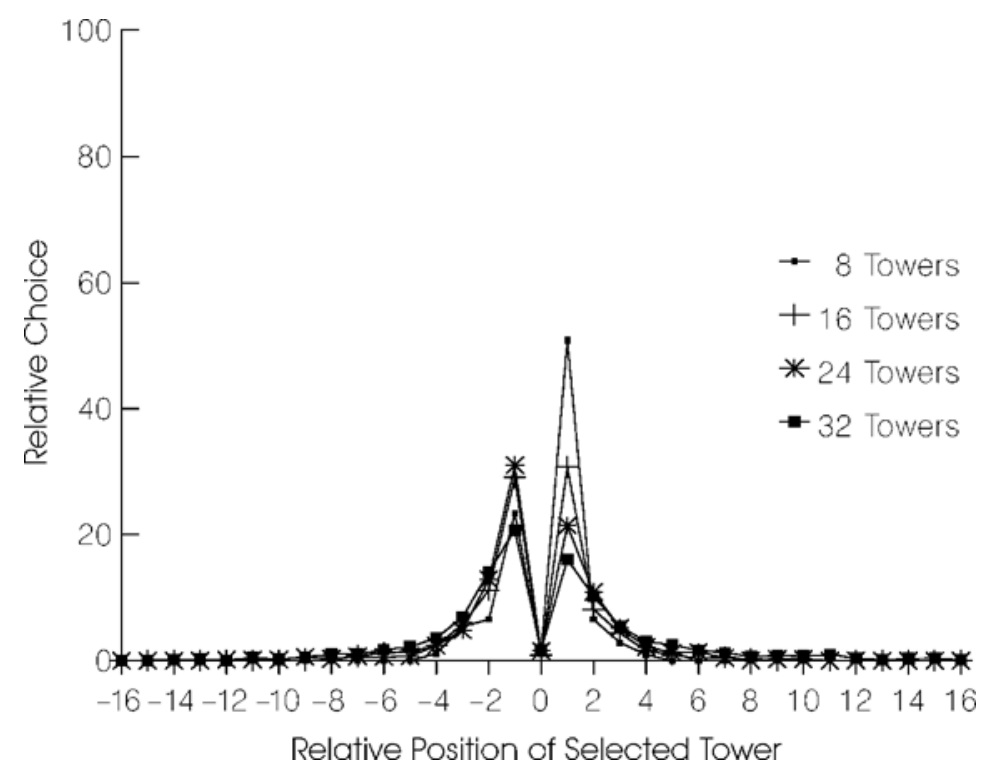

Figure 11. Frequency distributions of the number of towers for which choices are removed from the previous choice, with $8,16,24$, and 32 towers spaced $66 \mathrm{~cm}$ apart in Experiment 4. Negative numbers refer to counterclockwise shifts, whereas positive numbers refer to clockwise shifts.

such pairs, as well as a triplet of arms, in the 16-arm condition attracted more errors than would be expected by chance. The hypothesis that spatial separation between adjacent arms was even more important than the number of arms was tested formally in Experiment 2, with the number of arms and the spatial arrangement of those arms being varied systematically. The evidence obtained from Experiment 2 revealed that spatial arrangement was much more important than number of arms. When the arms were tightly grouped together, the centers of the entrances to the arms were only $10 \mathrm{~cm}$ apart, and performance was relatively poor regardless of number of arms. When the arms were as widely separated as the number added would permit, performance declined as more and more arms were added. However, in view of the lack of any differences in performance as arms were added in the tight configuration, the performance differences in the spreadout configurations were assumed to have been due to the decreasing separation between adjacent arms, rather than to the number of arms, as more and more arms were added.

One explanation for why decreasing the separation between adjacent arms might lead to poorer performance on a radial maze is that closely grouped arms are easily confused. It has often been shown (Olton \& Samuelson, 1976; Staddon, 1983; Suzuki, Augerinos, \& Black, 1980) that rats depend more on extramaze cues than on intramaze cues when solving a radial maze. Staddon suggested that rats "tag" each arm on the radial maze with both a spatial and a temporal code as they visit them. The spatial code identifies the arm with reference to some distinctive extramaze cue, whereas the temporal code is tied to the time of visit but then decays with pass- ing time from that point on. As Staddon pointed out, if rats locate and remember arms with respect to their location, using extramaze cues, such as pictures on the wall, doors, corners, and so on, this task should be easier when the arms are widely, as opposed to closely, spaced. When arms are mere millimeters apart at the central platform and the angular separation is only about $7^{\circ}$, it is probably difficult to relate these arms to the relatively sparse external cues in a typical laboratory. Actually, the tight condition would be easier to deal with if the rats used intramaze cues, such as second from the left or the one with the knot in the wood. However, as many studies have shown (e.g., Olton \& Samuelson, 1976; Zoladek \& Roberts, 1978), rats do not seem to make use of intramaze cues under normal circumstances.

Dale (1982) reported data consistent with Staddon's (1983) theory. He exposed one group of rats to a standard radial maze and two other groups, one of them blinded, to a parallel maze. The parallel maze consisted of eight parallel alleys, each $10 \mathrm{~cm}$ wide and $80 \mathrm{~cm}$ long, mounted on a platform $100 \times 93 \mathrm{~cm}$. Dale found that the rats performed much better on the radial maze than on the parallel maze. Moreover, he found that there was no difference between the performance of the sighted and the blind rats on the parallel maze. Dale took this as evidence that the rats were unable to use extramaze cues effectively on the parallel maze. At such a disadvantage were the sighted rats on this apparatus that they could not perform better than blind rats! His conclusion was that with the arms so close together, each arm could not be sufficiently distinguished from its neighbors on the basis of extramaze cues. Although the arms on the parallel maze were longer 
than those used in Experiments 1 and 2 of the present research and the separation between adjacent arms was even tighter, the two arrangements were similar.

Cohen, Burkhart, Jones, and Innis (1990) tested rats on a 16 -arm radial maze in which only 8 arms were open at any time. The floors of half the open arms were covered in hardware cloth, whereas those of the remaining 4 were left smooth. Also, in different conditions, the open arms were either grouped together or separated by closed arms. Although a direct comparison with the present research is difficult due to the use of forcedchoice procedures and the use of intramaze cues, one finding of interest is consistent with both Staddon's (1983) hypothesis and the present results. Cohen et al. reported that more errors were made when the arms were close together than when they were more widely separated.

Grobety and Schenk (1992) found increased errors on an eight-arm radial maze when three pairs of the arms were set close together instead of spaced equidistantly. They ruled out a confusion interpretation, however, because their rats tended to select only one arm in each adjacent pair during their first selections. In its place, they offered the explanation that rats have a predisposition to look far afield after any choice in a foraging situation. The results from Experiment 1 in the present research, however, are different from those reported by Grobety and Schenk and are consistent with the confusion hypothesis. The rats in Experiment 1, having visited one arm of a tightly clustered pair, tended next to visit the other arm in that pair instead of a more distant arm. One possible explanation for the discrepancy between our results and those reported by Grobety and Schenk is provided by optimal foraging theory (Charnov, 1976). That theory predicts that the larger central platform used in our experiment would make wide-ranging subsequent choices less likely, due to the energy cost that such wanderings would incur, than in the case of the research reported by Grobety and Schenk, who used a much smaller central platform.

In summary, the results of Experiments 1 and 2 confirmed that performance on the radial maze depended more on the spatial arrangement of food locations than it did on the number of locations per se. In particular, closely spaced arms were associated with a greater number of errors, and the inability to discriminate among closely spaced arms seems to afford the best explanation.

Experiments 3 and 4 were conducted to vary the number of food locations available to rats while holding the spatial separation between adjacent locations constant. This was accomplished by using towers standing on the floor, instead of arms on a radial maze, as the food locations. By expanding the diameter of the circle of food towers as more and more were added, it was possible to maintain a constant separation of $33 \mathrm{~cm}$ (Experiment 3 ) or $66 \mathrm{~cm}$ (Experiment 4) between the centers of the adjacent towers. This approach resulted in the percentage of correct choices declining, up to a point, as more and more towers were added. The results of Experiment 3 showed that the percentage of correct choices declined from 8 to 16 towers but did not decline beyond that. The results of Experiment 3 therefore suggested that under the conditions in effect, the limit of spatial memory for food locations in rats lies somewhere between 8 and 16 locations. However, mediocre performance in the 8-tower condition in Experiment 3 was interpreted as indicating that spatial separation might still have been affecting performance. A separation between adjacent towers of only $33 \mathrm{~cm}$ is much less than that between adjacent food locations on the typical radial maze. The adjacent food tower separation of $66 \mathrm{~cm}$ employed in Experiment 4 revealed declines in the percentage of correct choices as 8 towers were increased to 16 and, again, as 16 towers were increased to 24, but not beyond that. Because the percentage of novel choices in the first $n$ opportunities in the 8-tower condition in Experiment 4, at 85\% correct, was more typical of that found on the radial maze (Olton et al., 1977; Olton \& Samuelson, 1976), it was assumed that the 66-cm tower separation was sufficient to reduce the influence of spatial separation to the level typically seen in the radial maze literature. While it is possible that increasing the separation between adjacent towers still more might result in an even higher estimate, there are indications that such would not be the case. The effects of expanding the separation between adjacent towers in going from Experiment 3 to Experiment 4 were seen mostly in the 8 - and 16 -tower conditions, with very little change being observed in the 24- or 32-tower conditions. Accordingly, the data from Experiment 4 were assumed to have provided a better estimate of the limit of spatial memory for food locations in rats. To the extent that the results of Experiments 3 and 4 lend themselves to an explanation in terms of memory, they suggest that with a 66- $\mathrm{cm}$ separation between adjacent towers, the limit of spatial memory for food locations in rats is somewhere between 16 and 24. Consistent with this interpretation is the observation that in Experiment 3, the probability of a correct response on successive choices crossed the chance line in the 16-tower condition but did not clearly do so until the 24-tower condition in Experiment 4.

Both on a radial maze, such as that used in Experiments 1 and 2, and in the sort of food tower arrangements used in Experiments 3 and 4 of the present research, the opportunity to simply choose adjacent food sites in preference to more distant sites was always present. Such opportunities for patterned responding must be taken into account in interpreting the results of these studies in terms of memory.

In Experiment 2 of the present research, with the central platform $152 \mathrm{~cm}$ in diameter, the rats showed a strong tendency to choose adjacent arms in the eight-arm spreadout condition. However, as the arms got closer and closer together in that experiment, this tendency to visit adjacent arms changed to one of visiting every other arm as the rats circumvented the maze. One explanation for this finding is that the rats were unwilling to make the sharp turns that would be required to visit adjacent arms under tight conditions. Yoerg and Kamil (1982) noted a similar effect in 
their research and made a similar interpretation in terms of the minimal turning radius. Grobety and Schenk (1992), on the other hand, argued for an explanation in terms of a predisposition against looking for food close by where food has just been found. More research will be needed to choose between these different explanations for an effect that has now been reported a number of times.

The opportunity to simply circle the maze was an even greater concern in the Stonehenge arrangement than on the radial maze. In Experiments 3 and 4, with no arms and no central platform, the rats did not have to return to the center of the apparatus before making another choice. In such a setting, it was comparatively easy for the rats to simply make circuits of the arena, selecting adjacent towers one after the other, and they often did so. Indeed, circling is predicted by optimal foraging theory (Charnov, 1976). According to that theory, the cost in terms of energy expenditure of crossing an open arena to visit the tower directly opposite to that just abandoned is minimal when the arena is small but becomes quite costly as the diameter of the arena increases. Yoerg and Kamil (1982) found that rats showed an increasing tendency to choose adjacent arms as the central platform of a radial maze was increased in size. However, it is important to note that this change in strategy did not increase accuracy of choices. Hoffman, Timberlake, Leffel, and Gont (1999) tested rats on a variety of floor mazes consisting of runways laid flat on the floor so that the rats could step off if they chose to do so. These runways ran from a central platform to each of six food cups set on the floor (radial maze), connected the six food cups directly (circle maze), or did both (wheel and spoke maze). Regardless of the configuration, the rats tended to stay on the runways even when doing so meant that they had to travel further than they would have had they stepped off and traveled directly to a food cup. In another arrangement, one that closely resembled our own, Hoffman et al. removed the runways totally and had a circle of six food cups set on the floor surrounding a central platform. In this arrangement, the rats tended to go from cup to cup to such an extent that $49 \%$ of the visits were to adjacent cups. In Experiments 3 and 4 in the present research, consistent with Hoffman et al., the rats also showed a strong tendency to select adjacent towers (about 55\% averaged over all the conditions in both experiments). Presumably, this tendency toward the selection of adjacent towers in an open-field setting such as ours was because the towers, even in Experiment 3, were always wider apart than the midlines of the entrances to the arms had been in Experiment 2, except in the eightarm spread-out condition. In addition, sharp turns were not required to select adjacent towers in our Stonehenge arrangement (cf. Hoffman et al., 1999).

Regardless, once a circuiting rat starts skipping arms or towers, it is not clear that a circling strategy provides any advantage with respect to remembering novel locations. Most important of all, however, there is little evidence in the present data (cf. Yoerg \& Kamil, 1982) that such patterning affected recall of novel locations. The only significant change in the tendency to make +1 and -1 responses was seen when 24 towers were increased to 32 towers in Experiment 4, but this was not accompanied by a decrease in the percentage of correct responses. As a result, it does not seem likely that the orderly changes in the percentage of correct choices seen in Experiment 4 can be attributed to patterning strategies.

Another question raised by the use of a maze with a very large central platform, or one with food towers in an open arena, concerns the estimates of chance performance. Olton and Samuelson (1976) originally argued that the reason for selecting the radial maze for their research was that it made viable the assumption that on exiting any given arm, the rat was able to freely choose from among all the arms, including the one just exited. Since then, several writers have provided reasons to question the assumption that random sampling, with replacement, from $n$ arms is the best estimate of chance performance (cf. Eckerman, 1980; Lanke, Mansson, Bjerkemo, \& Kjellstrand, 1993). Eckerman examined the impact on chance performance, as revealed by Monte Carlo simulations, of various assumptions of postchoice response bias. His findings revealed estimates of chance performance ranging from $65 \%$ novel choices with no bias assumed, to $70 \%$ with the assumption that the arm just exited is not available on the next choice, to $72 \%$, assuming, in addition, that adjacent arms on either side of the just-exited arm are especially favored on the next choice. Clearly, to the extent that varying the size of the central platform or of an open arena affects the rate of selecting adjacent food locations, the assumption of $65 \%$ correct choices by chance becomes increasingly difficult to sustain. In Experiments 3 and 4, not only was the size of the arena varied, but also there were no arms to force the rats back to the middle of the arena. Thus, there was nothing to prevent the rats from simply selecting adjacent towers, and they did this frequently. Nevertheless, an assumption of random sampling, with replacement, was made throughout the present research because it has been the value most frequently used in related research. It is interesting to note that our estimate that the limit of spatial memory in rats under these conditions lies between 16 and 24 locations accords reasonably well with an estimate for the limit of spatial memory provided by Olton et al. (1977). Their estimate that the limit is between 25 and 30 locations was based on a statistical extrapolation of their own data to chance levels. However, their estimate might have to be reconsidered in the light of the data regarding chance performance on the radial maze reported by Eckerman. If chance levels were assumed to be higher-say, $70 \%$ or $72 \%$ - the estimate of the limit of spatial memory provided by Olton et al. would have to be revised downward, bringing it closer to the estimate provided herein. Our estimate, not being based entirely on assumptions of chance performance, is less subject to reconsideration as estimates of the value of chance performance are changed. 
This series of experiments suggests that the radial maze is not the best apparatus for testing the effect of number of food locations on spatial memory in rats. With any radial maze that has a central platform of fixed diameter, there will be an unavoidable confound between number of arms and the spatial separation of those arms. Olton and Samuelson (1976) used a central platform that was $34 \mathrm{~cm}$ in diameter; with 8 arms, each $86 \mathrm{~cm}$ long, attached to the maze, the separation between the midlines of adjacent arms at the platform was about $13 \mathrm{~cm}$, and the separation between adjacent food cups was about $81 \mathrm{~cm}$. In the case of Olton et al. (1977), the central platform was $87 \mathrm{~cm}$ in diameter; with $17 \mathrm{arms}$, each $69 \mathrm{~cm}$ long, on the maze, the midlines of the arms were about $16 \mathrm{~cm}$ apart where they joined the platform and were about $42 \mathrm{~cm}$ apart at the food cup ends. As was pointed out in Experiment 3, a radial maze would have to attain unwieldy proportions in order to support 48 well-separated arms, each $61 \mathrm{~cm}$ long. All in all, the food tower approach based on the work of Brown and Terrinoni (1996) seems to provide a much more suitable apparatus for studies in which the number of food locations for rats is varied.

In summary, the present series of experiments suggests that under the conditions examined, the limit of spatial memory in rats is between 16 and 24 locations. This estimate is considerably higher than Miller's (1956) estimate of the capacity of human working memory as being seven items plus or minus two. The fact that the capacity of spatial memory in rats appears to exceed that of symbolic memory in humans will likely not surprise those who have studied spatial memory in rats. The widespread consensus has been that rats have very good spatial memory. The ability to remember places visited or not visited has clear survival benefits for foraging and food-storing animals such as the rat.

\section{REFERENCES}

Bond, A. B., Cook, R. G., \& LAMB, M. R. (1981). Spatial memory and the performance of rats and pigeons in the radial-arm maze. Animal Learning \& Behavior, 9, 575-580.

Brown, M. F., \& Terrinoni, M. (1996). Control of choice by the spa- tial configuration of goals. Journal of Experimental Psychology: Animal Behavior Processes, 22, 438-446.

Charnov, E. L. (1976). Optimal foraging: The marginal value theorem. Theoretical Population Biology, 9, 129-136.

Cohen, J. S., Burkhart, P., Jones, N., \& InNIS, N. K. (1990). The effects of an intramaze cue search rule on rats' spatial working memory. Behavioural Processes, 22, 73-88.

DALE, R. H. I. (1982). Parallel-arm performance of sighted and blind rats: Spatial memory and maze structure. Behaviour Analysis Letters, 2, 127-139.

Dallal, N. L., \& Meck, W. H. (1990). Hierarchical structures: Chunking by food type facilitates spatial memory. Journal of Experimental Psychology: Animal Behavior Processes, 16, 69-84.

Eckerman, D. A. (1980). Monte Carlo estimation of chance performance for the radial arm maze. Bulletin of the Psychonomic Society, 15, 93-95.

Grobety, M.-C., \& Schenk, F. (1992). The influence of spatial irregularity upon radial-maze performance in the rat. Animal Learning \& Behavior, 20, 393-400.

Hoffman, C. M., Timberlake, W., Leffel, J., \& Gont, R. (1999). How is radial-arm maze behavior in rats related to locomotor search tactics? Animal Learning \& Behavior, 27, 426-444.

Lanke, J., Mansson, L., Bjerkemo, M., \& Kjellstrand, P. (1993). Spatial memory and stereotypic behaviour of animals in radial arm mazes. Brain Research, 605, 221-228.

Miller, G. A. (1956). The magical number seven plus or minus two: Some limits on our capacity for processing information. Psychological Review, 63, 81-97.

Olton, D. S., Collison, C., \& Werz, M. A. (1977). Spatial memory and radial arm maze performance of rats. Learning \& Motivation, $\mathbf{8}$, 289-314.

Olton, D. S., \& Samuelson, R. J. (1976). Remembrance of places passed: Spatial memory in rats. Journal of Experimental Psychology: Animal Behavior Processes, 2, 97-116.

Roberts, W. A. (1979). Spatial memory in the rat on a hierarchical maze. Learning \& Motivation, 10, 117-140.

STADDON, J. E. R. (1983). Memory and temporal control. In J. E. R. Staddon (Ed.), Adaptive behavior and learning (pp. 354-394). Cambridge: Cambridge University Press.

Suzuki, S., Augerinos, G., \& Black, A. H. (1980). Stimulus control of spatial behavior on the eight-arm maze in rats. Learning \& Motivation, 11, 1-18.

Tulving, E., \& Pearlstone, Z (1966). Availability versus accessibility of information in memory for words. Journal of Verbal Learning \& Verbal Behavior, 5, 381-391.

Yoerg, S. I., \& KAmiL, A. C. (1982). Response strategies in the radial arm maze: Running around in circles. Animal Learning \& Behavior, 10, 530-534.

ZoladeK, L., \& Roberts, W. A. (1978). The sensory basis of spatial memory in the rat. Animal Learning \& Behavior, 6, 77-81. 


\section{APPENDIX}

According to Olton and Samuelson (1976), the expected probability of being correct by chance alone (expressed as a percentage) on each of Choices 2 through $n$ is

$$
p(\text { cor }) \exp =\frac{\text { number of arms notyetchosen }}{\text { totalnumber of arms }} \times 100 .
$$

Following Bond et al. (1981), p(cor)exp may be calculated from the following formula:

$$
p(\text { cor }) \exp =[(n-1) / n]^{r-1} \times 100,
$$

where $n=$ the number of arms and $r=$ the number of the choice in question.

Thus, on an eight-arm maze, the $p$ (cor)exp is $(7 / 8)^{1}=88$ on Choice 2 and $(7 / 8)^{2}=77$ on Choice 3 . By Choice 8, $p$ (cor)exp is only 39. As a result, Olton and Samuelson (1976) calculated a corrected probability that their rats were correct on each of Trials 2 through $n$ as follows:

$$
\text { corrected } p(\text { cor }) \text { obs }=\frac{p(\text { cor }) \text { obs }-p(\text { cor }) \exp }{100-p(\text { cor }) \exp } \times 100
$$

where, $p$ (cor)obs referred to the actual observed probability that the rats were correct on successive choices. For example, with 5 rats over 10 trials, there are 50 instances of a second choice. The statistic $p$ (cor)obs would be the percentage of these 50 trials on which the rats were actually correct (i.e., they selected a novel arm). Something like a correlation coefficient, this statistic ranges from +100 to -100 .

When the statistic is +100 , it means that on a particular choice-say, Choice 5 -the rats were always correct. When the statistic is 0 , it means that the performance is at chance level, and when the statistic is below 0 , it means that performance is lower than chance. As an example, consider a group of 5 rats that, collectively, make 40 correct choices out of the 50 choices they make on their 5 th choice over 10 trials on an eight-arm maze:

$$
\text { corrected } p(\text { cor }) \text { jobs }=\frac{(40 / 50) \times 100-(7 / 8)^{4} \times 100}{100-(7 / 8)^{4} \times 100} \times 100=50.47 \text {. }
$$

Thus, being right 8 out of 10 times on Trial 5 is better than chance performance, but a long way from perfect. For a further discussion of this statistic, see Olton and Samuelson (1976).

(Manuscript received March 10, 2003;

revision accepted for publication July 23, 2003.) 